\title{
THE HEWITT REALCOMPACTIFICATION OF PRODUCTS
}

BY

\author{
HARUTO OHTA
}

\begin{abstract}
For a completely regular Hausdorff space $X, v X$ denotes the Hewitt realcompactification of $X$. Given a topological property $\mathscr{P}$ of spaces, our interest is in characterizing the class $R(\mathscr{P})$ of all spaces $X$ such that $v(X \times Y)=v X \times v Y$ holds for each $\mathscr{P}$-space $Y$. In the present paper, we obtain such characterizations in the case that $\mathscr{P}$ is locally compact and in the case that $\mathscr{P}$ is metrizable.
\end{abstract}

Introduction. All spaces considered in this paper are assumed to be completely regular Hausdorff and all maps are continuous. The Hewitt realcompactification $v X$ of a space $X$ is the unique realcompactification of $X$ to which each real-valued continuous function on $X$ admits a continuous extension. For details of Hewitt realcompactifications, the reader is referred to [8]. An important problem in the theory concerns when the relation $v(X \times Y)=v X \times v Y$ is valid. Following [23], [30], we denote by $\mathcal{R}$ (resp. $\mathcal{R}(\mathscr{P}))$ the class of all spaces $X$ such that $v(X \times Y)=$ $v X \times v Y$ holds for each space $Y$ (resp. each $\mathscr{P}$-space $Y$ ), where $\mathscr{P}$ is a given property of spaces. It is known that: (Comfort [4], [5]) a locally compact, realcompact space of nonmeasurable cardinal belongs to $R$; (Hušek [12], [14] and McArthur [23]) every member of $\Re$ is realcompact; (Hušek [13], [14]) every member of $\mathcal{R}$ is of nonmeasurable cardinal; [28] every member of $\mathcal{R}$ is locally compact. These facts characterize $R$ as precisely the class of locally compact, realcompact spaces of nonmeasurable cardinals. Further, in [30], the author has tried to characterize $\mathcal{R}(\mathscr{P})$ for various properties $\mathcal{P}$ of spaces, and has proved that $\Re=\mathscr{R}$ (metacompact) $=\mathscr{R}$ (subparacompact). It is the purpose of this paper to continue our study along this line, in particular, the following results are established:

(A) Both $\mathcal{R}$ (locally compact) and $\mathscr{R}$ (Moore) coincide with the class of all spaces of nonmeasurable cardinals whose Hewitt realcompactifications are locally compact.

(B) The class $R$ (metrizable) consists precisely of all weak cb*-spaces, in the sense of Isiwata [20], of nonmeasurable cardinals.

In $\S 1$, we present a technical theorem which is useful in finding a pair $X, Y$ of spaces for which $v(X \times Y)=v X \times v Y$ fails. Our result (A) is then proved. We also give a positive answer to the following question of Hušek [13, p. 326]: Do there

Received by the editors April 12, 1978 and, in revised form, October 24, 1978.

AMS (MOS) subject classifications (1970). Primary 54D60, 54B10; Secondary 54D45, 54E35.

Key words and phrases. Hewitt realcompactification, product space, locally compact space, metrizable space, weak $\mathrm{cb}^{*}$-space, pseudo-m-compact space, quotient map, perfect map, $C$-embedding, absolute, nonmeasurable cardinal, $D(\mathfrak{m})$-expandable family. 
exist minimal cardinals $\mathfrak{m}, \mathfrak{n}$ for which $|X|=\mathfrak{m},|Y|=\mathfrak{n}$ and $v(X \times Y) \neq v X \times$ $v Y$. In $\S 2$, we prove the analogue for $v X$ of the corollary to Glicksberg's theorem [9, Theorem 1]: For onto maps $f_{i}: X_{i} \rightarrow Y_{i}(i=1,2), \beta\left(Y_{1} \times Y_{2}\right)=\beta Y_{1} \times \beta Y_{2}$ holds whenever $\beta\left(X_{1} \times X_{2}\right)=\beta X_{1} \times \beta X_{2}$, where $\beta X$ is the Stone-Čech compactification of $X$. It is shown that some additional conditions must be imposed in order that the analogous " $v$ " theorem holds. In $\$ 3$, we apply our theory to prove (B), and also show that $\mathcal{R}$ (locally compact, metrizable) is precisely the class of all spaces of nonmeasurable cardinals. When studying the relation $v(X \times T)=v X \times v T$ with a metrizable factor $T$, the central issue is the weak $\mathrm{cb}^{*}$ property in another factor $X$. It is proved that, in case $X$ satisfies the countable chain condition and $T$ is metrizable, the relation holds if and only if (i) either $X$ or $T$ is of nonmeasurable cardinal and (ii) either $X$ is a weak $\mathrm{cb}^{*}$-space or $T$ is locally compact. Finally a number of problems are posed in $\$ 4$.

Throughout the paper, $\mathfrak{m}$ and $\mathfrak{n}$ denote cardinal numbers, and $\mathfrak{m}^{+}$denotes the smallest cardinal greater than $\mathrm{m}$. We let $w, d, c$ and $\chi$ denote the following cardinal functions: weight, density, cellularity and character (cf. [7]). $|A|$ denotes the cardinality of a set $A$, and $\mathrm{m}_{1}$ stands for the first measurable cardinal. Since $\mathrm{m}_{1}$ (if it exists) is greater than any nonmeasurable cardinal, that $|A|$ is nonmeasurable is denoted by $|A|<\mathfrak{m}_{1}$. We also denote by $C(X)$ the set of real-valued continuous functions on a space $X$. For general terminology, see [7] and [8].

1. Characterizations of $\mathcal{R}$ (locally compact) and $\mathcal{R}$ (Moore). Two subsets $A$ and $B$ of a space $X$ are said to be completely separated in $X$ if there is $f \in C(X)$ such that $f(A)=\{0\}$ and $f(B)=\{1\}$. A family $\left\{F_{\alpha}\right\}$ of subsets of a space $X$ is called expandable if there is a locally finite family $\left\{G_{\alpha}\right\}$ of open sets in $X$ with $F_{\alpha} \subset G_{\alpha}$ for each $\alpha$. We introduce a new class of expandable families.

1.1. Definition. A family $\left\{F_{\alpha} \mid \alpha \in A\right\}$ of subsets of a space $X$ is $D(\mathfrak{m})$-expandable if there exists a locally finite family $\left\{G_{\alpha} \mid \alpha \in A\right\}$ of open sets in $X$ with $F_{\alpha} \subset G_{\alpha}$ for each $\alpha \in A$ and each $F_{\alpha}$ is the union of at most $m$ subsets each of which is completely separated from $X-G_{\alpha}$.

If $\mathfrak{n} \geqslant \mathfrak{m}$, then a $D(\mathfrak{m})$-expandable family is $D(\mathfrak{n})$-expandable. As a space is completely regular Hausdorff, every expandable family in $X$ is $D(|X|)$-expandable, and a uniformly locally finite family defined in [17] is $D(1)$-expandable (cf. [27]). Recall from [17] that a space $X$ is pseudo-m-compact if each locally finite family of nonempty open sets in $X$ has cardinality less than $m$. Pseudocompact spaces are known to be precisely pseudo- $\boldsymbol{\aleph}_{0}$-compact spaces. The following theorem plays an essential role in our discussions.

1.2. Theorem. Let $X \times Y$ be $C$-embedded in $X \times v Y$. If there exists a $D(m)$-expandable family $\mathscr{F}$ in $Y$, with $|\mathcal{F}|=\mathfrak{n}$, such that $\cap\left\{\mathrm{cl}_{\nu Y} F \mid F \in \mathscr{F}\right\} \neq \varnothing$, then each point $x \in X$, with $\chi(x, X) \leqslant \mathrm{n}$, has a pseudo-m-compact neighborhood.

Proof. Suppose on the contrary that there exists a point $x_{0} \in X$, with $\chi\left(x_{0}, X\right)$ $\leqslant \mathrm{n}$, which has no pseudo-m-compact neighborhood. Let $\left\{G_{\lambda} \mid \lambda \in \Lambda\right\}$ be a neighborhood base at $x_{0}$ in $X$ with $|\Lambda|=\mathrm{n}$. Then, for each $\lambda \in \Lambda, \mathrm{cl}_{X} G_{\lambda}$ is not pseudo-m-compact, and thus there is a locally finite family $\left\{G_{\lambda \mu}^{\prime} \mid \mu \in M_{\lambda}\right\}$ of 
nonempty open sets in $\operatorname{cl}_{X} G_{\lambda}$ with $\left|M_{\lambda}\right|=\mathrm{m}$. Setting $G_{\lambda \mu}=G_{\lambda \mu}^{\prime} \cap G_{\lambda}$ for each $\mu \in M_{\lambda}$, we have a locally finite family $\left\{G_{\lambda \mu} \mid \mu \in M_{\lambda}\right\}$ of nonempty open sets in $X$. It can be assumed without loss of generality that $x_{0} \notin U\left\{G_{\lambda_{\mu}} \mid \mu \in M_{\lambda}\right\}$. For each $\mu \in M_{\lambda}$, pick $x_{\lambda \mu} \in G_{\lambda \mu}$, and choose $f_{\lambda \mu} \in C(X)$ such that $f_{\lambda \mu}\left(x_{\lambda \mu}\right)=0$ and $f_{\lambda \mu}\left(X-G_{\lambda \mu}\right)=\{1\}$. On the other hand, since $|\mathscr{F}|=\mathfrak{n}$, we may write $\mathscr{F}=$ $\left\{F_{\lambda} \mid \lambda \in \Lambda\right\}$. Then there is a locally finite family $\left\{H_{\lambda} \mid \lambda \in \Lambda\right\}$ of open sets in $Y$ with $F_{\lambda} \subset H_{\lambda}$ for each $\lambda \in \Lambda$. Each $F_{\lambda}$ is a union of $m$ subsets each of which is completely separated from $Y-H_{\lambda}$, and so we express it by $F_{\lambda}=$ $\cup\left\{F_{\lambda \mu} \mid \mu \in M_{\lambda}\right\}$, i.e., there is $g_{\lambda \mu} \in C(Y)$ such that $g_{\lambda \mu}\left(F_{\lambda \mu}\right)=\{0\}$ and $g_{\lambda \mu}\left(Y-H_{\lambda}\right)=\{1\}$. For each $\lambda \in \Lambda$ and each $\mu \in M_{\lambda}$, let us set

$$
\begin{aligned}
J_{\lambda \mu} & =\left\{x_{\lambda \mu}\right\} \times F_{\lambda \mu}, \quad K_{\lambda \mu}=G_{\lambda \mu} \times H_{\lambda}, \\
h_{\lambda \mu}((x, y)) & =\min \left\{1, f_{\lambda \mu}(x)+g_{\lambda \mu}(y)\right\}, \quad(x, y) \in X \times Y .
\end{aligned}
$$

Then $h_{\lambda \mu} \in C(X \times Y), h_{\lambda \mu}\left(J_{\lambda \mu}\right)=\{0\}$ and $h_{\lambda \mu}\left((X \times Y)-K_{\lambda \mu}\right)=\{1\}$. It is easily checked that $\mathscr{K}=\left\{K_{\lambda \mu} \mid \mu \in M_{\lambda}, \lambda \in \Lambda\right\}$ is locally finite in $X \times Y$. Therefore if we define a function $h$ on $X \times Y$ by

$$
h(p)=\inf \left\{h_{\lambda \mu}(p) \mid \mu \in M_{\lambda}, \lambda \in \Lambda\right\}, p \in X \times Y,
$$

then $h$ is continuous. Let us choose $y_{0} \in \cap\left\{\operatorname{cl}_{\nu Y} F_{\lambda} \mid \lambda \in \Lambda\right\}$; then $y_{0} \in v Y-Y$, because $\mathcal{F}$ is locally finite in $Y$. Now we show that $h$ admits no continuous extension to the point $p_{0}=\left(x_{0}, y_{0}\right) \in X \times v Y$. Let $U \times V$ be a given basic neighborhood of $p_{0}$ in $X \times v Y$. There is $\lambda \in \Lambda$ with $G_{\lambda} \subset U$, and $V \cap F_{\lambda \mu} \neq \varnothing$ for some $\mu \in M_{\lambda}$. Choose $y \in V \cap F_{\lambda \mu}$. Then both $p_{1}=\left(x_{\lambda \mu}, y\right)$ and $p_{2}=\left(x_{0}, y\right)$ belong to $U \times V$ and $h\left(p_{1}\right)=0$, while $h\left(p_{2}\right)=1$. This shows that $h$ does not extend continuously to $p_{0}$, which contradicts the assumption that $X \times Y$ is $C$-embedded in $X \times v Y$. Hence the proof is complete.

1.3. Corollary. Let $X \times Y$ be $C$-embedded in $X \times v Y$. If there exists a locally finite family $\mathcal{H}$ of nonempty open sets in $Y$, with $|\mathcal{H}|=\mathfrak{n}$, such that

$$
\bigcap\left\{\mathrm{cl}_{\nu Y} H \mid H \in \mathcal{H}\right\} \neq \varnothing,
$$

then each point $x \in X$, with $\chi(x, X) \leqslant \mathfrak{n}$, has a pseudo-c(Y)-compact neighborhood.

Proof. Let $\mathcal{H}=\left\{H_{\lambda} \mid \lambda \in \Lambda\right\}$, and choose $y_{0} \in \cap\left\{\operatorname{cl}_{\nu Y} H_{\lambda} \mid \lambda \in \Lambda\right\}$. For each $\lambda \in \Lambda$, by Zorn's lemma, there is a maximal disjoint family $\mathscr{F}_{\lambda}$ of nonempty open sets in $H_{\lambda}$ such that each $F \in \mathscr{F}_{\lambda}$ is completely separated from $Y-H_{\lambda}$. Let us set $F_{\lambda}=\cup\left\{F \mid F \in \mathscr{F}_{\lambda}\right\}$. For each $\lambda \in \Lambda$, the maximality of $\mathscr{F}_{\lambda}$ implies that $y_{0} \in$ $\mathrm{cl}_{\nu Y} F_{\lambda}$. Since $\left|\mathscr{F}_{\lambda}\right| \leqslant c(Y),\left\{F_{\lambda} \mid \lambda \in \Lambda\right\}$ is a $D(c(Y))$-expandable, family in $Y$, with $|\Lambda|=\mathfrak{n}$, such that $\cap\left\{\mathrm{cl}_{v Y} F_{\lambda} \mid \lambda \in \Lambda\right\} \neq \varnothing$. Thus the corollary follows from Theorem 1.2.

1.4. Remark. Let us say that a family $\mathcal{G}$ of subsets of a space $X$ converges to $x \in X$ if each neighborhood of $x$ contains some member of $\mathcal{G}$, and that a subspace $S$ of $X$ is relatively pseudo-m-compact in $X$ if each locally finite family $U$ of nonempty open sets in $X$ such that $S \cap U \neq \varnothing$ for each $U \in \mathcal{Q}$ has cardinality less than $\mathrm{m}$. The conclusion of Theorem 1.2 (resp. Corollary 1.3) can be generalized 
as follows: Each convergent family $\mathcal{G}$ of subsets of $X$, with $|\mathcal{G}| \leqslant \mathfrak{n}$, has a member which is relatively pseudo-m-compact (resp. relatively pseudo- $c(Y)$-compact) in $X$.

Our next work is to construct spaces $Y$ which have a $D\left(\aleph_{0}\right)$-expandable family $\mathscr{F}$ such that $\cap\left\{\operatorname{cl}_{\nu Y} F \mid F \in \mathscr{F}\right\} \neq \varnothing$. A space is called 0 -dimensional if it has a base consisting of open-and-closed sets. For an ordinal $\alpha$, we denote by $W(\alpha)$ the set of all ordinals less than $\alpha$ topologized with the order topology, and by $\omega_{0}$ (resp. $\omega_{1}$ ) the first infinite (resp. first uncountable) ordinal.

1.5. FACT. For every infinite cardinal $\mathfrak{n}$, there exists a 0-dimensional locally compact space $Y=Y_{1}(\mathfrak{n})$, with $|Y|=w(Y)=\mathfrak{n} \cdot \boldsymbol{\aleph}_{1}$, that has a $D\left(\boldsymbol{\aleph}_{0}\right)$-expandable family $\mathscr{F}$ such that $|\mathscr{F}|=\mathrm{n}$ and $\cap\left\{\operatorname{cl}_{\nu} F \mid F \in \mathscr{F}\right\} \neq \varnothing$.

Proof. Let $T_{1}=W\left(\omega_{1}+1\right) \times W\left(\omega_{0}+1\right)$, and let $T_{2}=\Lambda \cup\{\infty\}$ be the one point compactification of a discrete space $\Lambda$ of cardinality $\mathfrak{n}$. We denote a base for the topology on $T_{i}$ by $\Re_{i}$ for $i=1,2$. Let $E=\left\{\left(\omega_{1}, \beta\right) \mid \beta<\omega_{0}\right\}$, and let $Z^{\prime}$ be the quotient space obtained from $R=T_{1} \times T_{2}$ by collapsing the set $\left\{\left(\omega_{1}, \beta\right)\right\} \times T_{2}$ to a point $z(\beta) \in Z^{\prime}$ for each $\left(\omega_{1}, \beta\right) \in E$. Let $\phi^{\prime}: R \rightarrow Z^{\prime}$ be the quotient map. Let $Z_{0}$ be the set $Z^{\prime}$, retopologized by letting $\cup\left\{\Re_{(B)} \mid B \in \mathscr{B}_{1}\right\}$ be a base, where

$$
\mathscr{B}(B)=\left\{\begin{array}{l}
\left\{\phi^{\prime}\left(B \times T_{2}\right)\right\} \quad \text { if } B \cap E \neq \varnothing, \\
\left\{\phi^{\prime}\left(B \times B^{\prime}\right) \mid B^{\prime} \in \mathscr{B}_{2}\right\} \quad \text { if } B \cap E=\varnothing .
\end{array}\right.
$$

Then the natural map $\phi: R \rightarrow Z_{0}$ is continuous, and hence $Z_{0}$ is compact. Let us set

$$
Z=Z_{0}-\phi\left(\left\{\left(\left(\gamma, \omega_{0}\right), \infty\right) \mid \gamma \leqslant \omega_{1}\right\}\right) .
$$

The space $Z$ is a 0 -dimensional locally compact space with $|Z|=w(Z)=\mathfrak{n} \cdot \aleph_{1}$. Since $z(\beta)$ is a $P$-point for each $\beta<\omega_{0}$, it is easily checked that $Z$ is $C$-embedded in $Z \cup\left\{z_{0}\right\}$, where $z_{0}=z\left(\omega_{0}\right)$, and so $z_{0} \in v Z-Z$ by $[8,8.6]$. Setting $D_{\lambda}=$ $\phi\left(\left\{\left(\left(\gamma, \omega_{0}\right), \lambda\right) \mid \gamma<\omega_{1}\right\}\right)$ for each $\lambda \in \Lambda$, we have a discrete family $\left\{D_{\lambda} \mid \lambda \in \Lambda\right\}$ of closed subsets in $Z$ such that $z_{0} \in \cap\left\{\operatorname{cl}_{v Z} D_{\lambda} \mid \lambda \in \Lambda\right\} \neq \varnothing$. Define a subspace $Y$ of the product space $Z \times W\left(\omega_{0}+1\right)$ by

$$
Y=\left(Z \times\left\{\omega_{0}\right\}\right) \cup\left(\cup\left\{D_{\lambda} \times W\left(\omega_{0}+1\right) \mid \lambda \in \Lambda\right\}\right) .
$$

Then $Y$ is a 0 -dimensional locally compact space with $|Y|=w(Y)=\mathfrak{n} \cdot \aleph_{1}$, because $Y$ is a closed subspace of $Z \times W\left(\omega_{0}+1\right)$. It remains to show the existence of a $D\left(\boldsymbol{\aleph}_{0}\right)$-expandable family in $Y$ satisfying the stated conditions. Since $Z \times\left\{\omega_{0}\right\}$ is $C$-embedded in $Y$, it follows from $[8,8.10(a)]$ that $v Z=v\left(Z \times\left\{\omega_{0}\right\}\right) \subset v Y$, and hence we may consider $z_{0}$ as an element of $v Y-Y$. Setting $F_{\lambda}=D_{\lambda} \times W\left(\omega_{0}\right)$ for each $\lambda \in \Lambda$, we have a discrete family $\mathscr{F}=\left\{F_{\lambda} \mid \lambda \in \Lambda\right\}$ of open sets in $Y$ such that $z_{0} \in \cap\left\{\operatorname{cl}_{\nu Y} F_{\lambda} \mid \lambda \in \Lambda\right\}$. Then, since each $F_{\lambda}$ is a union of countably many open-and-closed subsets in $Y, \mathscr{F}$ is a $D\left(\aleph_{0}\right)$-expandable family in $Y$. Hence $Y$ is proved to be the desired space.

1.6. FACT. For every infinite cardinal $\mathfrak{n}$, there exists a 0 -dimensional Moore space $Y=Y_{2}(\mathfrak{n})$, with $|Y|=w(Y)=\mathfrak{n} \cdot \exp \aleph_{0}$, that has a $D\left(\aleph_{0}\right)$-expandable family $\mathscr{F}$ such that $|\mathcal{F}|=\mathfrak{n}$ and $\cap\left\{\operatorname{cl}_{\nu} F \mid F \in \mathscr{F}\right\} \neq \varnothing$. 
Proof. In [30], for every infinite cardinal $\mathfrak{n}$, we constructed a 0 -dimensional Moore space $Z=Z(\mathfrak{n})$, with $|Z|=w(Z)=\mathfrak{n} \cdot \exp \aleph_{0}$, that has a discrete family $\mathscr{D}$ of closed subsets such that $|\mathscr{D}|=\mathfrak{n}$ and $\cap\left\{\operatorname{cl}_{v Z} D \mid D \in \mathscr{D}\right\} \neq \varnothing$. The desired space $Y_{2}(\mathfrak{n})$ can be made from $Z(\mathfrak{n})$ by the same procedure as in the proof of 1.4.

For later use, we quote a theorem due to Hušek [13]:

1.7. TheOREM (HuŠEK). Let $Q$ be a discrete space. Then $v(P \times Q)=v P \times v Q$ holds if and only if either $|P|<\mathrm{m}_{1}$ or $|Q|<\mathrm{m}_{1}$ (i.e., either $|P|$ or $|Q|$ is nonmeasurable).

We are now in a position to prove main theorems of this section. For the notion of locally pseudocompact spaces see [5]. We remark that the assumption $|X|<\mathrm{m}_{1}$ of Theorem 1.8 is useful only for the implications (a) $\rightarrow$ (b) and (a) $\rightarrow$ (c).

1.8. THEOREM. The following conditions on a space $X$ with $|X|<m_{1}$ are equivalent:

(a) $X$ is locally pseudocompact.

(b) $X \times Y$ is $C$-embedded in $X \times v Y$ for each 0 -dimensional locally compact space $Y$ with $w(Y) \leqslant \chi(X) \cdot \aleph_{1}$.

(c) $X \times Y$ is $C$-embedded in $X \times v Y$ for each 0 -dimensional Moore space $Y$ with $w(Y) \leqslant \chi(X) \cdot \exp \aleph_{0}$.

Proof. We proved in [28] that if $X$ is a locally pseudocompact space of nonmeasurable cardinal, then $X \times Y$ is $C$-embedded in $X \times v Y$ for each $k$-space $Y$. Since both locally compact spaces and Moore spaces are $k$-spaces, (a) $\rightarrow(b)$ and (a) $\rightarrow$ (c) follow from this result. To prove (b) $\rightarrow$ (a) ((c) $\rightarrow$ (a)) suppose on the contrary that $X$ is not locally pseudocompact at $x_{0} \in X$. Let $Y$ be the space $Y_{1}(\mathfrak{n})$ $\left(Y_{2}(\mathfrak{n})\right)$ constructed in $1.5(1.6)$, where $\mathfrak{n}=\chi\left(x_{0}, X\right)$. Then it follows from Theorem 1.2 , that $X \times Y$ is not $C$-embedded in $X \times v Y$. This contradiction completes the proof.

1.9. THEOREM. The following conditions on a space $X$ are equivalent:

(a) $v X$ is locally compact and $|X|<\mathrm{m}_{1}$.

(b) $v(X \times Y)=v X \times v Y$ holds for each 0-dimensional locally compact space $Y$ with $w(Y) \leqslant \chi(v X) \cdot \aleph_{1}$.

(c) $v(X \times Y)=v X \times v Y$ holds for each 0-dimensional Moore space $Y$ with $w(Y) \leqslant \chi(v X) \cdot \exp \kappa_{0}$.

Proof. Since $(a) \rightarrow(b)$ and $(a) \rightarrow$ (c) follow from Hušek [14, Corollary (a), p. 177] (cf. also [28]), we prove only (b) $\rightarrow$ (a) and (c) $\rightarrow$ (a). By Theorem 1.8, $v X$ is locally pseudocompact, and hence it is locally compact, because every pseudocompact realcompact space is compact [7, 3.11.1]. Suppose that $|X|>\mathfrak{m}_{1}$; then $\chi(v X)$ $\geqslant m_{1}$ by [21, Theorem 2]. If we take for $Y$ a discrete space of cardinality $m_{1}$, then it follows from Theorem 1.7 that $v(X \times Y) \neq v X \times v Y$. This contradicts (b) and (c) simultaneously. Hence the proof is complete.

In [14], Hušek proved that if $X$ satisfies 1.9(a), then $v(X \times Y)=v X \times v Y$ holds for each $k$-space $Y$. Therefore Theorem 1.9 tells us that both $\mathcal{R}$ (locally compact) 
and $R$ (Moore) coincide with the class of spaces $X$ such that $v X$ is locally compact and $|X|<\mathfrak{m}_{1}$.

1.10. Remarks. (1) Let $\Psi$ be the space described in [8, 5I, p. 79]; $\Psi$ is constructed as follows: Let $\mathcal{E}$ be a maximal infinite almost-disjoint family of infinite subsets of the set $N$ of integers. Then $|\mathcal{E}|=\exp \aleph_{0}$. The space $\Psi$ is the union of $N$ with a new set $D=\left\{\omega_{E} \mid E \in \mathcal{E}\right\}$ of distinct points endowed with the following topology: Each point of $N$ is isolated, and a neighborhood of $\omega_{E}$ is any set containing $\omega_{E}$ and all but a finite number of points of $E$. It is well known that $\Psi$ is a 0 -dimensional pseudocompact (and hence $\beta \Psi=v \Psi$ by [8, 8A4, p. 125]) locally compact Moore space. In [25], Mrówka showed that $\mathcal{E}$ can be chosen so that $\beta \Psi$ is the one point compactification. Then, dividing $D$ into a disjoint family of countable infinite subsets, we have a discrete family $\mathscr{D}$ of closed subsets in $\Psi$ such that $|\mathscr{D}|=\exp \aleph_{0}$ and $\cap\left\{\operatorname{cl}_{v \Psi} D^{\prime} \mid D^{\prime} \in \mathscr{D}\right\} \neq \varnothing$. Thus, by the same method as in the proof of 1.5 , we can make a 0-dimensional locally compact Moore space $Y$, with $w(Y)=$ $\exp \aleph_{0}$, that has a $D\left(\aleph_{0}\right)$-expandable family $\mathscr{F}$ such that $|\mathscr{F}|=\exp \aleph_{0}$ and $\cap\left\{\mathrm{cl}_{\nu Y} F \mid F \in \mathscr{F}\right\} \neq \varnothing$. This fact combined with Theorem 1.2 implies that the following condition (d) is also equivalent to 1.9(a) under the assumption that $\chi(v X) \leqslant \exp \aleph_{0}$.

(d) $v(X \times Y)=v X \times v Y$ holds for each 0-dimensional locally compact Moore space $Y$ with $w(Y) \leqslant \chi(v X) \cdot \exp \aleph_{0}$.

We do not know whether (d) implies 1.9(a) or not in general.

(2) We can apply our theory to answer the following question of Hušek [13, p. 326]: Do there exist spaces $X, Y$ of cardinalities $\kappa_{0}$ and $\kappa_{1}$, respectively, such that $v(X \times Y) \neq v X \times v Y$ ? Let $X$ be the space of rational numbers with the usual topology. We take for $Y$ the space $Y_{1}\left(\kappa_{0}\right)$ constructed in 1.4. Then $|X|=w(X)=$ $\aleph_{0}$ and $|Y|=w(Y)=\aleph_{1}$. Since $X$ is not locally pseudocompact, it follows from Theorem 1.2 that $v(X \times Y) \neq v X \times v Y$.

2. Mapping theorems. In this section, we give mapping theorems which will be used in the next section. As is well known, for a map $f: X \rightarrow Y$, there exists a continuous extension $v f: v X \rightarrow v Y$ of $f\left[8,8\right.$.7]. If $f_{i}: X_{i} \rightarrow Y_{i}$ is a map for $i=1,2$, then the product map $f=f_{1} \times f_{2}$ from $X_{1} \times X_{2}$ to $Y_{1} \times Y_{2}$ is defined by $f\left(\left(x_{1}, x_{2}\right)\right)$ $=\left(f_{1}\left(x_{1}\right), f_{2}\left(x_{2}\right)\right)$ for $\left(x_{1}, x_{2}\right) \in X_{1} \times X_{2}$.

2.1. TheOrem. Let $f_{i}: X_{i} \rightarrow Y_{i}(i=1,2)$ be onto maps. If $v f_{1} \times v f_{2}$ is a quotient map from $v X_{1} \times v X_{2}$ onto $v Y_{1} \times v Y_{2}$, then $v\left(X_{1} \times X_{2}\right)=v X_{1} \times v X_{2}$ implies $v\left(Y_{1} \times Y_{2}\right)=v Y_{1} \times v Y_{2}$.

More precisely, we have the following theorem:

2.2. Theorem. Let $F_{i}: X_{i}^{*} \rightarrow Y_{i}^{*}(i=1,2)$ be onto maps such that $F=F_{1} \times F_{2}$ is a quotient map, and let $X_{i}$ (resp. $Y_{i}=F_{i}\left(X_{i}\right)$ ) be a dense $C$-embedded subspace of $X_{i}^{*}$ (resp. $Y_{i}^{*}$ ). If $X_{1} \times X_{2}$ is $C$-embedded in $X_{1}^{*} \times X_{2}^{*}$, then $Y_{1} \times Y_{2}$ is $C$-embedded in $Y_{1}^{*} \times Y_{2}^{*}$.

Proof. Let us set $f_{i}=F_{i} \mid X_{i}(i=1,2)$ and $f=f_{1} \times f_{2}$. To show that $Y_{1} \times Y_{2}$ is $C$-embedded in $Y_{1}^{*} \times Y_{2}^{*}$, let $g \in C\left(Y_{1} \times Y_{2}\right)$. Since $h=g \circ f \in C\left(X_{1} \times X_{2}\right)$, by 
our assumption, there exists $H \in C\left(X_{1}^{*} \times X_{2}^{*}\right)$ such that $H \mid\left(X_{1} \times X_{2}\right)=h$. We shall show that (*) $H$ takes on the constant value $t_{p}$ on $F^{-1}(p)$ for each $p \in Y_{1}^{*} \times$ $Y_{2}^{*}$. Let $x \in X_{1}$; then $h(x, \cdot)=g\left(f_{1}(x), \cdot\right) \circ f_{2}$, where $h(x, \cdot)=h \mid\left(\{x\} \times X_{2}\right)$. Since $g\left(f_{1}(x), \cdot\right) \in C\left(Y_{2}\right)$, it has a continuous extension $G_{x}$ over $Y_{2}^{*}$. Then, $X_{2}$ being dense in $X_{2}^{*}, H(x, \cdot)=G_{x} \circ F_{2}$. Hence it follows that $H(x, \cdot)$ is constant on $\{x\} \times F_{2}^{-1}(y)$ for each $y \in Y_{2}^{*}$. This implies that $H$ is constant on $f_{1}^{-1}\left(y_{1}\right) \times$ $F_{2}^{-1}\left(y_{2}\right)$ for each $\left(y_{1}, y_{2}\right) \in Y_{1} \times Y_{2}^{*}$. Similarly, $H$ is constant on $F_{1}^{-1}\left(y_{1}\right) \times$ $f_{2}^{-1}\left(y_{2}\right)$ for each $\left(y_{1}, y_{2}\right) \in Y_{1}^{*} \times Y_{2}$. To see (*), let $p=\left(y_{1}, y_{2}\right) \in Y_{1}^{*} \times Y_{2}^{*}$. Then it follows from these facts that

$$
\begin{aligned}
& H(x, \cdot)=H\left(x^{\prime}, \cdot\right) \quad \text { for each } x, x^{\prime} \in F_{1}^{-1}\left(y_{1}\right), \\
& H(\cdot, x)=H\left(\cdot, x^{\prime}\right) \quad \text { for each } x, x^{\prime} \in F_{2}^{-1}\left(y_{2}\right),
\end{aligned}
$$

and from which (*) is proved. Define a function $G$ on $Y_{1}^{*} \times Y_{2}^{*}$ by $G(p)=t_{p}$ for each $p \in Y_{1}^{*} \times Y_{2}^{*}$. Then $H=G \circ F$ and $G \mid\left(Y_{1} \times Y_{2}\right)=g$. Since $F$ is a quotient map and $H$ is continuous, it follows from [7, 2.4.2] that $G$ is continuous. Hence our proof is complete.

Theorem 2.2 remains true if " $C$-embedded" is replaced by " $C$ "-embedded". Ishii proved in [18] that if $f: X \rightarrow Y$ is an open perfect onto map, then so is $v f$. This leads to the following corollary of Theorem 2.1 .

2.3. Corollary. If $f_{i}: X_{i} \rightarrow Y_{i}$ is an open perfect map onto $Y_{i}$ for $i=1,2$, then $v\left(X_{1} \times X_{2}\right)=v X_{1} \times v X_{2}$ implies $v\left(Y_{1} \times Y_{2}\right)=v Y_{1} \times v Y_{2}$.

The following theorem shows that in Theorem 2.1 the assumption that $v f_{1} \times v f_{2}$ is quotient onto cannot be dropped, even when $f_{1}$ is an identity and $f_{2}$ is a perfect map. Recall from [13] that a space $X$ is pseudo- $m_{1}$-compact if the cardinality of each locally finite family of nonempty open sets in $X$ is nonmeasurable.

2.4. TheOrem. Among the following conditions on a space $X,(\mathrm{a}) \rightarrow(\mathrm{b}) \rightarrow(\mathrm{c})$ is valid. Conversely, (c) $\rightarrow$ (a) holds if $|X|<\mathrm{m}_{1}$.

(a) $v X$ is locally compact.

(b) For each space $Y$ satisfying $v(X \times Y)=v X \times v Y$ and each quotient image $Z$ of $Y, v(X \times Z)=v X \times v Z$ holds.

(c) As in (b), with "perfect" instead of "quotient".

Proof. (a) $\rightarrow$ (b). Let $Y$ be a space satisfying $v(X \times Y)=v X \times v Y$, and let $Z$ be the image of $Y$ under a quotient map $f$. Since $v X$ is locally compact, by Whitehead's theorem [7, 3.3.17], $\mathrm{id}_{v X} \times f$ is a quotient map, where $\mathrm{id}_{v X}$ is the identity map of $v X$. It follows from Theorem 2.2 that $X \times Z$ is $C$-embedded in $v X \times Z$. Hušek proved in [13] that if $P$ is a locally compact, realcompact space, then $v(P \times Q)=v P \times v Q$ if and only if either $|P|<m_{1}$ or $Q$ is pseudo- $m_{1}$-compact. If we apply this theorem to our case, then $|v X|<m_{1}$ or $Y$ is pseudo- $m_{1}$-compact. If $Y$ is pseudo- $m_{1}$-compact, so is $Z$. Hence it follows that $v(v X \times Z)=v X \times$ $v Z$. Thus we have $v(X \times Z)=v X \times v Z$.

(b) $\rightarrow$ (c). Obvious.

(c) $\rightarrow$ (a). Suppose that $|X|<m_{1}$ and $v X$ is not locally compact at $x_{0} \in v X$. 
Then, by [7, 3.11.1], $x_{0}$ has no pseudocompact neighborhood in $v X$. Let $\mathfrak{n}=$ $\max \left\{|v X|, \chi\left(x_{0}, v X\right)\right\}$; then $\mathfrak{n}<m_{1}$. Let $\omega_{\alpha}$ be the initial ordinal of $\mathfrak{n}^{+}$, and let $T=W\left(\omega_{\alpha}+1\right) \times W\left(\omega_{0}+1\right)$. Let $\Lambda$ be a discrete space of cardinality $\mathfrak{n}$, and let $S_{0}$ be the quotient space obtained from $R_{0}=T \times \Lambda$ by collapsing the set $\left\{\left(\omega_{\alpha}, \beta\right)\right\} \times$ $\Lambda$ to a point $s(\beta)$ for each $\beta \in E$, where $E=\left\{2 n \mid n<\omega_{0}\right\} \cup\left\{\omega_{0}\right\}$. Let $g: R_{0} \rightarrow S_{0}$ be the quotient map. Let us set $S=S_{0}-\left\{s_{0}\right\}$, where $s_{0}=s\left(\omega_{0}\right)$, and let $R=R_{0}-$ $g^{-1}\left(s_{0}\right)$. Then it is easily checked that $v S=S_{0}$ and $v R=R_{0}$. If we set

$$
G=g\left(\left\{(\gamma, 2 n) \mid \gamma \leqslant \omega_{\alpha}, n<\omega_{0}\right\} \times \Lambda\right),
$$

then $G$ is a cozero-set of $S_{0}$, and hence $G=G^{*} \cap S_{0}$ for some cozero-set $G^{*}$ of $\beta S_{0}(=\beta S)$. Let us set $Z=S \cup G^{*}$. We now need the following lemma:

2.5. Lemma. Let $X \supset X_{1} \supset X_{2}$. Suppose that $X_{2}$ is dense in $X$ and is $C$-embedded in $X_{1}$. Then, for each open set $H$ of $X, X_{2} \cup H$ is $C$-embedded in $X_{1} \cup H$.

The proof is left to the reader, since it requires only routine verification. We continue the proof of Theorem 2.4. By Lemma 2.5 and [7, 3.11.10], $v Z=S_{0} \cup G^{*}$, and hence $s_{0} \in v Z-Z$. Setting

$$
F_{\lambda}=g\left(\left\{(\gamma, 2 n+1) \mid \gamma \leqslant \omega_{\alpha}, n<\omega_{0}\right\} \times\{\lambda\}\right)
$$

for each $\lambda \in \Lambda$, we obtain a locally finite family $\left\{F_{\lambda} \mid \lambda \in \Lambda\right\}$ of open sets in $Z$. Since each $F_{\lambda}$ is a countable union of open-and-closed subsets of $Z,\left\{F_{\lambda} \mid \lambda \in \Lambda\right\}$ is a $D\left(\boldsymbol{\aleph}_{0}\right)$-expandable family in $Z$ such that $\cap\left\{\mathrm{cl}_{v Z} F_{\lambda} \mid \lambda \in \Lambda\right\} \ni s_{0}$. Since $\chi\left(x_{0}, v X\right)$ $\leqslant|\Lambda|$, it follows from Theorem 1.2 that $v(X \times Z) \neq v X \times v Z$. For our end, it suffices to show that $Z$ is the perfect image of a space $Y$ satisfying $v(X \times Y)=v X$ $\times v Y$. There exists the extension $\beta g: \beta R_{0} \rightarrow \beta S_{0}$ of $g$. Let us set $Y=R \cup H^{*}$, where $H^{*}=(\beta g)^{-1}\left(G^{*}\right)$, and set $f=(\beta g) \mid Y$. Then, since $H^{*}$ is a cozero-set of $\beta R_{0}$ $(=\beta R), v Y=R_{0} \cup H^{*}$ by Lemma 2.5 and [7, 3.11.10]. Further it is easily checked that $f$ is a perfect map from $Y$ onto $Z$ and $Y$ is locally compact. Since $|Y|<\mathrm{m}_{1}$, it follows from [5, Theorem 2.1] that $X \times Y$ is $C$-embedded in $v X \times Y$. It remains to show that $v X \times Y$ is $C$-embedded in $v X \times v Y$. Since $|v X| \leqslant \mathrm{n}$, a similar argument to that of $[8,8.20]$ shows that $v X \times W\left(\omega_{\alpha}\right) \times W\left(\omega_{0}+1\right)$ is $C$-embedded in $v X \times$ $W\left(\omega_{\alpha}+1\right) \times W\left(\omega_{0}+1\right)$. Thus $v X \times R$ is $C$-embedded in $v X \times R_{0}$. Since $v X \times$ $H^{*}$ is an open set of $v X \times \beta Y$, it follows from Lemma 2.5 that

$$
(v X \times R) \cup\left(v X \times H^{*}\right) \quad(=v X \times Y)
$$

is $C$-embedded in

$$
\left(v X \times R_{0}\right) \cup\left(v X \times H^{*}\right) \quad(=v X \times v Y) .
$$

Hence the proof is complete.

2.6. REMARK. In case $|X| \geqslant \mathrm{m}_{1}$, (c) $\rightarrow$ (a) of Theorem 2.4 need not be true. If $D$ is a discrete space of cardinality $m_{1}$, then, by Theorem 1.6, $D$ satisfies $2.4(\mathrm{c})$. But it is known [5, p. 115] that $v D$ is not even a $k$-space.

The following corollary is proved by using Theorem 2.4 repeatedly.

2.7. Corollary. Let $f_{i}: X_{i} \rightarrow Y_{i}(i=1,2)$ be quotient onto maps. If both $v X_{1}$ and $v Y_{2}$ are locally compact, then $v\left(X_{1} \times X_{2}\right)=v X_{1} \times v X_{2}$ implies $v\left(Y_{1} \times Y_{2}\right)=v Y_{1}$ $\times v Y_{2}$. 
3. Characterizations of $\mathcal{R}$ (metrizable). A space $X$ is called a weak $c b^{*}$-space if for each decreasing sequence $\left\{F_{n} \mid n<\omega_{0}\right\}$ of regular closed sets in $X$ with empty intersection, $\cap\left\{\mathrm{cl}_{v X} F_{n} \mid n<\omega_{0}\right\}=\varnothing$ holds, where a regular closed set is the closure of an open set. This notion was introduced by Isiwata [20] as a common generalization of realcompact spaces and weak cb-spaces in the sense of Mack and Johnson [22]. Since normal countably paracompact spaces, extremally disconnected spaces [8, 1H, p. 22] and pseudocompact spaces (or more generally, $M^{\prime}$-spaces in the sense of Isiwata [19]) are weak cb-spaces, they are weak $\mathrm{cb}^{*}$ spaces. In this section, we prove the following theorem:

3.1. THEOREM. The following conditions on a space $X$ are equivalent:

(a) $X$ is a weak $c b^{*}$-space and $|X|<\mathfrak{m}_{1}$.

(b) $v(X \times T)=v X \times v T$ holds for each metrizable space $T$.

(c) $v\left(X \times D(d(X))^{\omega}\right)=v X \times v D(d(X))^{\omega}$.

Here, $D(d(X))^{\omega}$ denotes the product of countably many copies of a discrete space of cardinality $d(X)$. Associated with each space $X$, there exist an extremally disconnected space $E(X)$ and a perfect irreducible map (i.e, a perfect map which takes proper closed subsets onto proper subsets) $e_{X}$ from $E(X)$ onto $X$. The space $E(X)$ is unique up to homeomorphism and is called the absolute of $X$ (cf. [16], [31]). To prove Theorem 3.1, we make use of the following lemmas. The next lemma follows immediately from [10, Theorem 2.4] and [11, Proposition 1.2]; the first part also appears in [15].

3.2. Lemma. A space $X$ is a weak $c b^{*}$-space if and only if $v E(X)=E(v X)$ holds. Moreover, in case $v E(X)=E(v X)$, then $e_{v X}$ is the extension of $e_{X}$ over $v E(X)$.

3.3. LEMMA [29]. Let $X$ be a space and $T$ a metrizable space. If either $X$ is extremally disconnected or $T$ is locally compact, then $X \times T$ is z-embedded in $\beta X \times T$ (i.e., each zero-set of $X \times T$ is the restriction to $X \times T$ of a zero-set of $\beta X \times T)$.

The next lemma is a corollary of Blair [1, Theorem 7.6]:

3.4. Lemma (Blair). Let $X \times Y$ be $z$-embedded in $\beta X \times Y$. If either $|X|<\mathfrak{m}_{1}$ or $Y$ is pseudo- $m_{1}$-compact, then $v(X \times Y)=v X \times v Y$ holds.

Proof of Theorem 3.1. (a) $\rightarrow$ (b). Let $X$ be a weak cb*-space with $|X|<m_{1}$ and $T$ a metrizable space. Since $|E(X)|<\mathrm{m}_{1}$, it follows from Lemmas 3.3 and 3.4 that $v(E(X) \times T)=v E(X) \times v T$. By Lemma $3.2 v e_{X}\left(=e_{v X}\right)$ is a perfect map from $v E(X)$ onto $v X$, and so $v e_{X} \times \mathrm{id}_{v T}$ is perfect. Hence it follows from Theorem 2.1 that $v(X \times T)=v X \times v T$.

(b) $\rightarrow$ (c). Obvious.

(c) $\rightarrow$ (a). Suppose on the contrary that $X$ is not a weak cb*-space. Then there is a locally finite sequence $\left\{G_{n} \mid n<\omega_{0}\right\}$ of open sets in $X$ with $\cap\left\{\operatorname{cl}_{v X} G_{n} \mid n<\omega_{0}\right\} \neq$ $\varnothing$. Since $c(X) \leqslant d(X)$, each point of $D(d(X))^{\omega}$ has no pseudo-c(X)-compact neighborhood, and $\chi\left(D(d(X))^{\omega}\right)=\aleph_{0}$. Hence it follows from Corollary 1.3 that $X \times D(d(X))^{\omega}$ is not $C$-embedded in $v X \times D(d(X))^{\omega}$. This contradicts (c). To 
prove that $|X|<\mathfrak{m}_{1}$, find a discrete family $\left\{G_{\alpha} \mid \alpha \in A\right\}$ of nonempty open sets in $D(d(X))^{\omega}$ with $|A|=d(X)$. Pick $t_{\alpha} \in G_{\alpha}$ for each $\alpha \in A$, and set $D=\left\{t_{\alpha} \mid \alpha \in A\right\}$. Then it is easily checked that $X \times D$ is $C$-embedded in $X \times D(d(X))^{\omega}$. Since $v D \subset v D(d(X))^{\omega},(\mathrm{c})$ implies $v(X \times D)=v X \times v D$. Hence it follows from Theorem 1.6 that $|X|<\mathfrak{m}_{1}$ or $|D|(=d(X))<\mathfrak{m}_{1}$. If $|D|<\mathfrak{m}_{1}$, then $|X|<\mathfrak{m}_{1}$ by [8, 12.5]. Thus the proof is complete.

3.5. Remarks. (1) If $E(X) \times T$ is $z$-embedded in $\beta E(X) \times T$, then it follows from [6, Proposition 5.1] and [2, Corollary 3.6] that $E(X) \times T$ is $C$-embedded in $v E(X) \times T$. Therefore the proof of Theorem 3.1 shows that, more generally, a space $X$ is a weak cb*-space if and only if $X \times T$ is $C$-embedded in $v X \times T$ for each metrizable space $T$.

(2) The product $X \times T$ of a weak $\mathrm{cb}^{*}$-space $X$ with a metrizable space $T$ need not be $z$-embedded in $\beta X \times T$. In fact, it was remarked in [29] that $\operatorname{dim}(X \times T)$ $\leqslant \operatorname{dim} X+\operatorname{dim} T$ whenever $X \times T$ is $z$-embedded in $\beta X \times T$, while Wage showed in [32] that there exist a Lindelöf space (hence a weak cb*-space) $X$ and a metrizable space $T$ such that $\operatorname{dim}(X \times T)>\operatorname{dim} X+\operatorname{dim} T$.

(3) Lemmas 3.3 and 3.4 can be combined with Theorem 1.6 to yield the following result: $v(X \times T)=v X \times v T$ holds for each locally compact, metrizable space $T$ if and only if $|X|<\mathrm{m}_{1}$.

We conclude this section with a theorem, which gives conditions on $X$ and $Y$ necessary and sufficient that the relation $v(X \times Y)=v X \times v Y$ be valid in a restrictive situation.

3.6. THEOREM. Let $X$ be a space satisfying the countable chain condition (i.e., $\left.c(X) \leqslant \aleph_{0}\right)$ and $T$ a metrizable space. Then $v(X \times T)=v X \times v T$ holds if and only if (i) either $|X|<\mathfrak{m}_{1}$ or $|T|<\mathfrak{m}_{1}$ and (ii) either $X$ is weak $c b^{*}$ or $T$ is locally compact.

Proof. Necessity: (i) is proved just like (c) $\rightarrow$ (a) in Theorem 3.1. If $T$ is not locally compact, then $T$ is not locally pseudocompact by [7, 3.10.21 and 4.1.17]. Thus it follows from Corollary 1.3 that $X$ is a weak $\mathrm{cb}^{*}$-space.

Sufficiency: In case $|X|<m_{1}$, then the proof follows from Theorem 3.1 and Lemmas 3.3 and 3.4. In case $|T|<\mathfrak{m}_{1}$, then $T$ is realcompact by [8, 15.20]. It follows from [5, Corollary 2.2] and 3.5(1) that $v(X \times T)=v X \times v T$. Hence the proof is complete.

3.7. Remark. Theorem 3.6 fails to be valid if we drop the assumption $c(X)<\aleph_{0}$. To see this, we utilize the space $Q$ of all rational numbers and the space $Y_{0}$ due to Comfort [4, p. 99]. The space $Y_{0}$ was constructed as the quotient space obtained from the product space

$$
Z=W\left(\omega_{0}\right) \times W\left(\omega_{1}+1\right) \times W\left(\omega_{1}+1\right)
$$

by identifying, for each $n<\omega_{0}$ and each $\gamma \leqslant \omega_{1}$, the two points $\left(n, \omega_{1}, \gamma\right)$ and $\left(n+1, \gamma, \omega_{1}\right)$. Let $f: Z \rightarrow Y_{0}$ be the quotient map, and let us set $X=Y_{0}-\left\{y_{0}\right\}$, where $y_{0}$ is the center point $f\left(\left(0, \omega_{1}, \omega_{1}\right)\right)\left(=f\left(\left(n, \omega_{1}, \omega_{1}\right)\right)\right)$. Then he proved that $v X=Y_{0}$, and a similar argument assures us that $v(X \times Q)=v X \times Q$. Obviously $Q$ is metrizable but not locally compact. It remains to show that $X$ is not a weak 
cb*-space. Setting

$$
F_{n}=f\left(\{i \mid i \geqslant n\} \times W\left(\omega_{1}+1\right) \times W\left(\omega_{1}+1\right)\right) \cap X
$$

for each $n<\omega_{0}$, we obtain a decreasing sequence $\left\{F_{n} \mid n<\omega_{0}\right\}$ of regular closed sets in $X$ with empty intersection. Then $y_{0} \in \cap\left\{\operatorname{cl}_{v X} G_{n} \mid n<\omega_{0}\right\}$, and hence $X$ is not a weak $\mathrm{cb}^{*}$-space.

4. Problems and remarks. Many interesting problems related to our results remain unsolved. Following [20], we say that a space $X$ is $v$-locally compact if $v X$ is locally compact.

4.1. Characterize $\mathcal{R}(v$-locally compact). It is easy to see that

$$
\Re(v \text {-locally compact })=\Re(\text { pseudocompact }) \text {. }
$$

4.2. Characterize $\Re$ (realcompact). Hušek [12], [14] and McArthur [23] proved that each member $X$ of this class, with $|X|<\mathfrak{m}_{1}$, is realcompact; however, the characterization is not yet known in complete form.

4.3. Characterize $\Re\left(\right.$ weak $\left.\mathrm{cb}^{*}\right)$. We note that it follows from Lemma 3.2 and Theorem 2.1 that $\mathcal{R}$ (weak $\left.\mathrm{cb}^{*}\right)=\mathscr{R}$ (extremally disconnected). Moreover, since

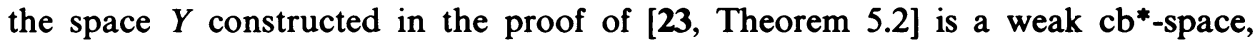
every member of $R$ (weak $\mathrm{cb}^{*}$ ) is realcompact.

4.4. Find conditions on $X$ and $T$ necessary and sufficient that $v(X \times T)=v X$ $\times v T$ be valid in the case where $T$ is a metrizable space.

4.5. Let $f_{i}: X_{i} \rightarrow Y_{i}(i=1,2)$ be onto maps. When does $v\left(Y_{1} \times Y_{2}\right)=v Y_{1} \times v Y_{2}$ imply $v\left(X_{1} \times X_{2}\right)=v X_{1} \times v X_{2}$ ?

4.6. RemarK. Let $f: Y \rightarrow Z$ be a perfect onto map. Then $v(X \times Z)=v X \times v Z$ does not necessarily imply $v(X \times Y)=v X \times v Y$, even when $\operatorname{id}_{v X} \times v f$ is a quotient onto map and $v X$ is compact. To see this, let us set $X=W\left(\omega_{1}\right)$; then by [23, Theorem 5.5] there exists a realcompact space $Y_{1}$ such that $v\left(X \times Y_{1}\right) \neq v X \times$ $v Y_{1}$. By [26, Corollary 2.3], $Y_{1}$ can be embedded as a closed subspace of a pseudocompact space $Y_{2}$. Let $i: Y_{1} \rightarrow Y_{2}$ be the embedding. Let us set $Y=Y_{1} \oplus$ $Y_{2}$ and $Z=Y_{2}$, where $\oplus$ means the topological sum. Define a map $f: Y \rightarrow Z$ by $f(y)=i(y)$ if $y \in Y_{1}$ and $f(y)=y$ if $y \in Y_{2}$. Then $f$ is a perfect onto map and $f f$ is a quotient map from $v Y\left(=Y_{1} \oplus v Y_{2}\right)$ onto $v Z\left(=v Y_{2}\right)$. Since $X$ is locally compact, it follows from [7, 3.10.26] that $X \times Z$ is pseudocompact, and hence

$$
v(X \times Z)=v X \times v Z
$$

holds by Glicksberg's theorem [9]. On the other hand, $v(X \times Y) \neq v X \times v Y$ obviously. Further, $v X$ being compact, it follows from [7, 3.3.17] that $\mathrm{id}_{v X} \times$ vf is a quotient onto map.

4.7. Find characterizations of an onto map $f: X \rightarrow Y$ for which $v f: v X \rightarrow v Y$ is an onto biquotient map in the sense of Michael [24]. We are interested in this problem in view of 4.8(3) below.

4.8. REMARK. It seems that the classes $\mathscr{R}(\mathcal{P})$ considered above have several common properties. Finally, we list some of these below. Each assertion follows from the results in the bracket. 
(1) $\mathscr{R}(\mathscr{P})$ includes all locally compact, realcompact spaces of nonmeasurable cardinals [5, Corollary 2.2].

(2) $\mathscr{R}(\mathscr{P})$ is closed under cozero-subspaces [3, 3.2].

(3) $R(\mathscr{P})$ is closed under open perfect images (Corollary 2.3); more generally, if $v f: v X \rightarrow v Y$ is an onto biquotient map, then $Y \in \mathcal{R}(\mathscr{P})$ whenever $X \in \mathcal{R}(\mathcal{P})$ ([24, Theorem 1.2] and Theorem 2.1).

(4) If each $\mathscr{P}$-space is $v$-locally compact, then $R(\mathscr{P})$ is closed under quotient images (Theorem 2.4).

(5) If $X \in \Re(\mathscr{P})$ and $Y$ is a locally compact, realcompact space with $|Y|<\mathrm{m}_{1}$, then $X \times Y \in \mathcal{R}(\mathcal{P})$ [5, Corollary 2.2].

\section{REFERENCES}

1. R. L. Blair, On v-embedded sets in topological spaces, TOPO-72, Lecture Notes in Math., vol. 378, Springer-Verlag, Berlin and New York, 1974, pp. 46-79.

2. R. L. Blair and A. W. Hager, Extensions of zero-sets and of real-valued functions, Math. Z. 136 (1974), 41-52.

3. N Notes on the Hewitt realcompactification of a product, General Topology Appl. 5 (1975), $1-8$.

4. W. W. Comfort, Locally compact realcompactifications, General Topology and its Relations to Modern Analysis and Algebra. II, Proc. Second Prague Topology Sympos., 1966, pp. 95-100.

5 . 107-118.

6. W. W. Comfort and S. Negrepontis, Extending continuous functions on $X \times Y$ to subsets of $\beta X \times \beta Y$, Fund. Math. 59 (1966), 1-12.

7. R. Engelking, General topology, Polish Scientific Publishers, Warsaw, 1977.

8. L. Gillman and M. Jerison, Rings of continuous functions, Van Nostrand, Princeton, N. J., 1960.

9. I. Glicksberg, Stone-Cech compactifications of products, Trans. Amer. Math. Soc. 90 (1959), 369-382.

10. K. Hardy and I. Juhász, Normality and weak cb property, Pacific J. Math. 64 (1976), 167-172.

11. K. Hardy and R. G. Woods, On c-realcompact spaces and locally bounded normal functions, Pacific J. Math. 43 (1972), 647-656.

12. M. Hušek, The Hewitt realcompactification of a product, Comment. Math. Univ. Carolin. 11 (1970), 393-395.

13. __ Pseudo-m-compactness and $v(P \times Q)$, Indag. Math. 33 (1971), 320-326.

14. Realcompactness of function spaces and $v(P \times Q)$, General Topology Appl. 2 (1972), $165-179$.

15. Y. Ikeda, Mappings and c-realcompact spaces, Bull. Tokyo Gakugei Univ. (4) 28 (1976), 12-16.

16. S. Iliadis and S. Fomin, The methods of centered systems in the theory of topological spaces, Russian Math. Surveys 21 (1966), 37-62.

17. J. R. Isbell, Uniform spaces, Math. Surveys, no. 12, Amer. Math. Soc., Providence, R. I., 1964.

18. T. Ishii, On the completions of maps, Proc. Japan Acad. Ser. A Math. Sci. 50 (1974), 39-43.

19. T. Isiwata, Generalizations of M-spaces. I, II, Proc. Japan Acad. Ser. A Math. Sci. 45 (1969), 359-363, 364-367.

20. __,$d-, d^{*}$-maps and $\mathrm{cb}^{*}$-spaces, Bull. Tokyo Gakugei Univ. (4) 29 (1977), 19-52.

21. I. Juhász, On closed discrete subspaces of product spaces, Bull. Acad. Polon. Sci. Sér. Sci. Math. Astronom. Phys. 17 (1969), 219-223.

22. J. Mack and D. G. Johnson, The Dedekind completion of $C(X)$, Pacific J. Math. 20 (1967), 231-243.

23. W. G. McArthur, Hewitt realcompactifications of products, Canad. J. Math. 22 (1970), 645-656.

24. E. Michael, Bi-quotient maps and cartesian products of quotient maps, Ann. Inst. Fourier (Grenoble) 18 (1968), 287-302.

25. S. G. Mrowka, Set theoretic constructions in topology, Fund. Math. 94 (1977), 83-92.

26. N. Noble, Countably compact and pseudocompact products, Czechoslovak Math. J. 19 (1969), 390-397. 
27. H. Ohta, Topologically complete spaces and perfect maps, Tsukuba J. Math. 1 (1977), 77-89.

28. __ Local compactness and Hewitt realcompactifications of products, Proc. Amer. Math. Soc. 69 (1978), 339-343.

29. S__ Some new characterizations of metrizable spaces (to appear).

30. L_ Local compactness and Hewitt realcompactifications of products. II (to appear).

31. D. P. Strauss, Extremally disconnected spaces, Proc. Amer. Math. Soc. 18 (1967), 305-309.

32. M. Wage, An easy counterexample to the inequality $\operatorname{dim}(X \times Y)<\operatorname{dim} X+\operatorname{dim} Y$ (to appear).

Institute of Mathematics, University of Tsukuba, Sakuramura Ibaraki, 300-31 Japan

Current address: Faculty of Education, Shizuoka University, Ohya, Shizuoka, 422 Japan 